\title{
Recurrent phosphaturic mesenchymal tumour of the temporal bone causing deafness and facial nerve palsy
}

\author{
M I SYED ${ }^{1}$, M CHATZIMICHALIS ${ }^{2}$, M RÖSSLE ${ }^{3}$, A M HUBER ${ }^{2}$ \\ ${ }^{1}$ Department of Otolaryngology, The Royal Infirmary, Edinburgh, Scotland, UK, ${ }^{2}$ Department of Otolaryngology \\ and ${ }^{3}$ Institute of Clinical Pathology, University Hospital Zurich, Switzerland
}

\begin{abstract}
Objective: We describe the first reported case of a phosphaturic mesenchymal tumour, mixed connective tissue variant, invading the temporal bone.

Case report: A female patient presented with increasing deafness. On examination there appeared to be a mass behind an intact tympanic membrane. Further radiological investigation showed a vascular mass occupying the middle ear, mastoid and internal auditory meatus. This was surgically resected and revealed to be a benign phosphaturic mesenchymal tumour, mixed connective tissue variant. The tumour recurred a year later, presenting as facial nerve palsy. A revision procedure was carried out; the tumour was excised with the sacrifice of a segment of the facial nerve, and a facial-hypoglossal nerve anastomosis was performed.

Conclusion: This case report highlights the occurrence of this benign but sometimes aggressive tumour, of which both clinicians and pathologists should be aware. Early recognition of the condition remains of utmost importance to minimise the debilitating consequences of long-term osteomalacia in affected patients, and to prevent extracranial and intracranial complications caused by the tumour.
\end{abstract}

Key words: Temporal Bone; Ear, Middle; Facial Paralysis; Malignant Mesenchymal Tumor; Oncogenic Osteomalacia

\section{Introduction}

The phosphaturic mesenchymal tumour, mixed connective tissue variant, represents an extremely rare, distinctive tumour that is frequently associated with oncogenic osteomalacia. Expression of fibroblast growth factor-23, a recently described protein putatively implicated in renal tubular phosphate loss, has been shown in a number of mesenchymal tumours with oncogenic osteomalacia. Oncogenic osteomalacia is a paraneoplastic syndrome wherein the presence of a tumour, often of mesenchymal origin, results in phosphate wasting and hypophosphataemia. It is thought that the tumour cells produce a peptide hormone like substance, identified as fibroblast growth factor-23, which is a physiological regulator of phosphate balance.

There are over a hundred cases of phosphaturic mesenchymal tumour, mixed connective tissue variant, described in literature. The tumours mainly involve the axial skeleton, the soft tissues and the paranasal sinuses. The vast majority of these tumours are benign, and recurrence and metastasis are rare even in the malignant cases. A significant number of these reported cases had initially been wrongly diagnosed; the misdiagnosis was detected by subsequent expert reviews.

The phosphaturic mesenchymal tumour, mixed connective tissue variant, remains a largely unknown entity, which continues to be misdiagnosed by most clinicians and pathologists.
We describe the first reported case of the tumour invading the temporal bone with intracranial extension. This led to deafness and facial paralysis, even after resection of the tumour with a subtotal petrosectomy. The diagnostic and management dilemmas are discussed.

\section{Case report}

A 71-year-old female patient presented with hearing loss in the right ear, which for 12 months had been gradually worsening. She had a history of congenital deafness in the left ear of unknown cause. She was also a type II diabetic and suffered from depression.

On examination of the patient's left ear, a reddish mass was visualised behind an intact tympanic membrane (Figure 1). The rest of the ear, nose and throat examination, including cranial nerve examination, was unremarkable. An audiogram showed moderate sensorineural hearing loss in the left ear. A magnetic resonance imaging (MRI) scan showed an enhancing vascular mass, which occupied the right middle ear and mastoid, extended to the lateral third of the internal auditory meatus, and encased the tympanic segment of the right internal carotid artery (Figure 2).

The patient subsequently underwent a tympanotomy. Pathomorphological examination of the tumour showed a haemangiopericytoma-like pattern with a moderately cellular proliferation of bland, round to oval cells, often arranged 


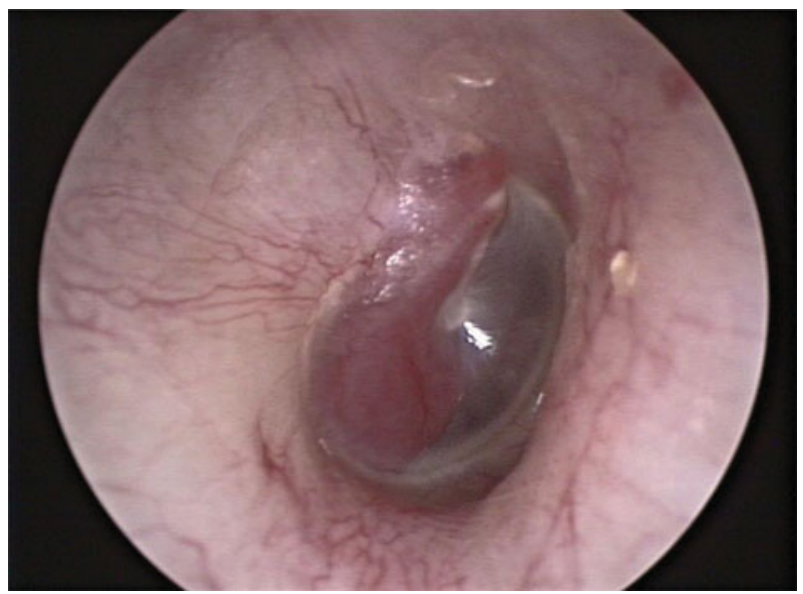

FIG. 1

Examination of the patient's left ear showed a reddish mass in the middle ear behind an intact tympanic membrane.

around thick-walled vessels. Due to the presence of typical 'grungy' calcification and a chondroid-like matrix in some areas, a histological diagnosis of mesenchymal phosphaturic tumour was made (Figure 3a). The patient had no clinical or metabolic signs of osteomalacia.

The patient underwent a petrosectomy with complete removal of the tumour. The tumour was extremely vascular, occupying the middle ear and mastoid, eroding into the lining of the sigmoid sinus, and invading the middle fossa dura, which had to be excised and primarily repaired. The macroscopic appearance of the tumour completely filling the mastoid cavity can be seen in Figure 4. Although the facial canal was intact, the tumour had to be peeled off the area surrounding the geniculate ganglion. The patient was advised post-operative radiotherapy, which she refused.

The patient presented a year later with a progressive, House-Brackmann grade IV, infranuclear facial nerve palsy on the right side. A repeated MRI scan showed recurrence of the tumour in the area of the genu and the cerebellopontine angle. A revision procedure was carried out and the tumour was macroscopically removed, with the sacrifice of a segment of the facial nerve at the genu. A facial-hypoglossal nerve anastomosis was carried out. The pathology examination confirmed the tumour to be a benign mesenchymal phosphaturic tumour, with infiltration of the peripheral nerves (Figure $3 b$ ).

\section{Discussion}

In 1987, Weidner and Santa Cruz published the first comprehensive study of mesenchymal tumours associated with oncogenic osteomalacia, and coined the term 'phosphaturic mesenchymal tumour, mixed connective tissue variant'. As part of this study, they reviewed previous cases published under a variety of diagnoses and reclassified them as a phosphaturic mesenchymal tumour, mixed connective tissue variant. However, as Folpe et $a l^{2}{ }^{2}$ demonstrated in 2004, this entity is still relatively unknown to both clinicians and pathologists, and continues to be misdiagnosed. (Folpe et $a .^{2}$ reviewed 32 mesenchymal tumours and concluded that more than half of these had been wrongly diagnosed.)

Phosphaturic mesenchymal tumours are rare neoplasms often described as 'strange tumours that occur in strange places'. Approximately 53 per cent have been reported

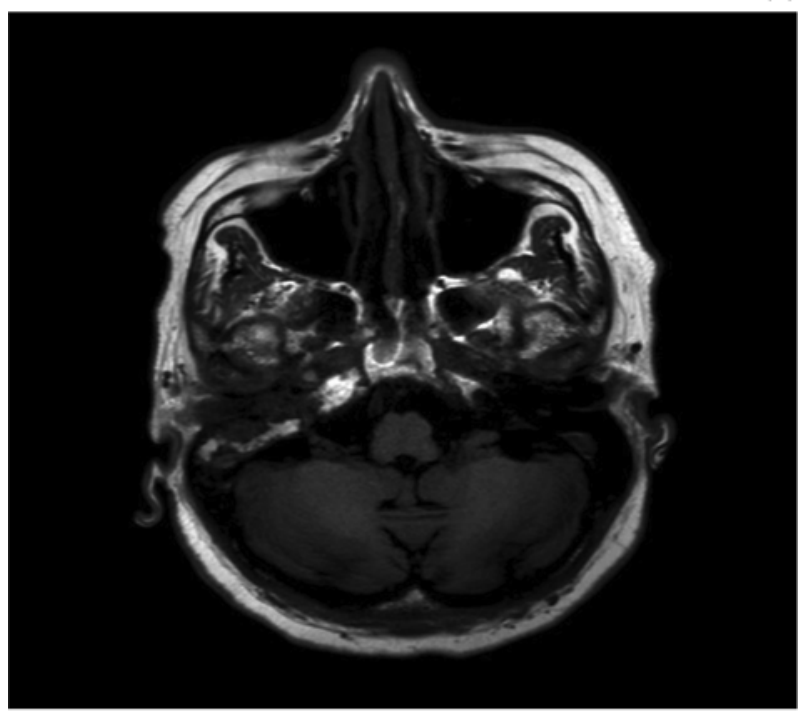

(b)

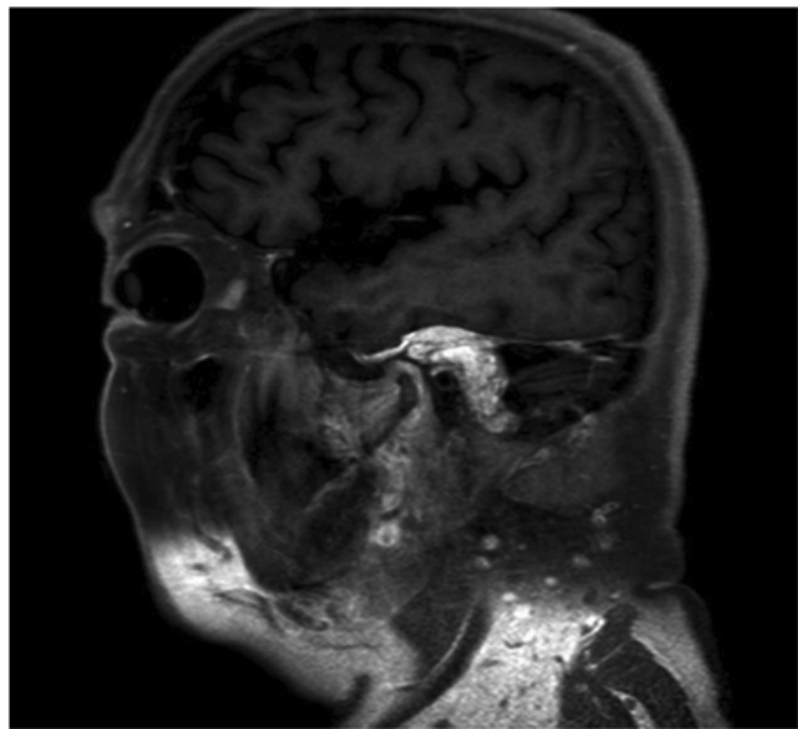

FIG. 2

(a) Axial and (b) sagittal magnetic resonance images show the enhancing mass occupying the middle ear, mastoid and petrous temporal bone.

occurring in bone, 45 per cent in soft tissues and 3 per cent in skin. In less than 5 per cent of cases, the tumours have been localised to the craniofacial region, involving the paranasal sinuses and mandible. Our case is the first that reports localisation of the tumour in the temporal bone, with intracranial extension.

Most osteomalacia-associated mesenchymal tumours have been found to express fibroblast growth factor-23, as confirmed in the review by Folpe et al., ${ }^{2}$ and by other investigators using immunohistochemistry, reverse transcription polymerase chain reaction, western blot and in-situ hybridisation techniques. ${ }^{3}$ Fibroblast growth factor-23 inhibits sodium-phosphate co-transporters in the proximal tubular cells of the kidney in vitro (via a decrease in the sodium phosphate transporter-type 2a (NPT2a) transporters). ${ }^{4}$ It has also been shown to inhibit 25-hydroxyvitamin $\mathrm{D}$ to 1,25-dihydroxyvitamin D. Furthermore, mice injected with 
(a)

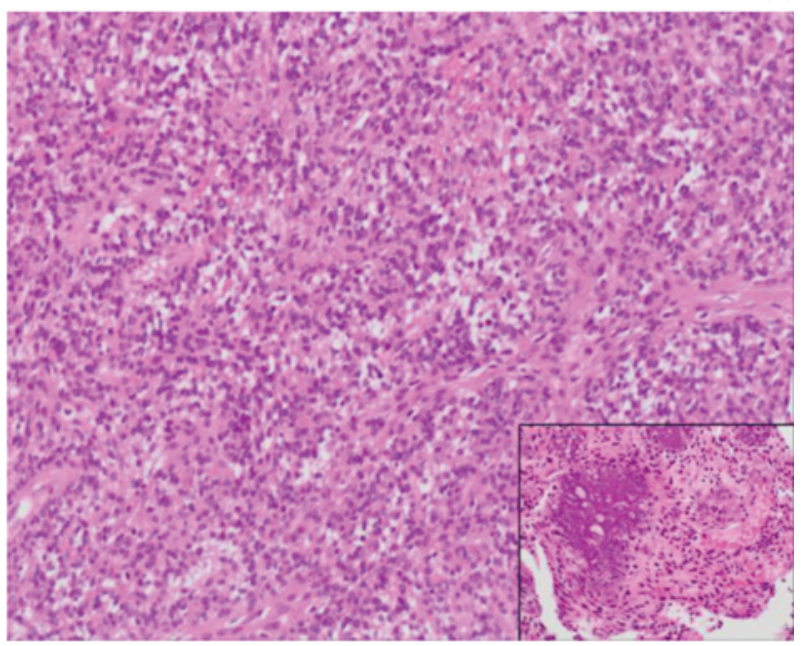

(b)

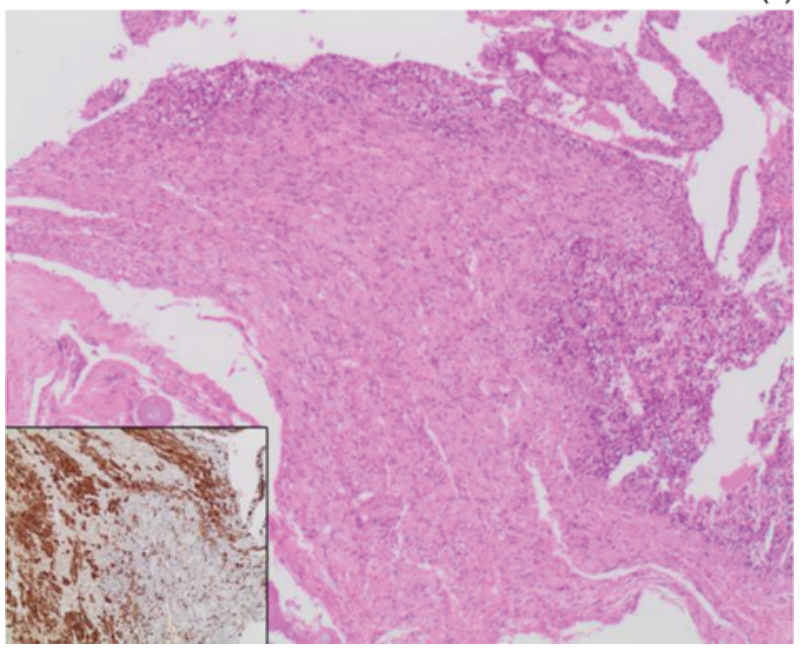

FIG. 3

Photomicrographs showing (a) the tumour (H\&E; 20X) (inset shows typical 'grungy' calcifications (H\&E; 10X)) and (b) infiltration of cells of the recurrent tumour in peripheral nerve tissue (H\&E; $2 \mathrm{X}$ ) (inset shows the immunohistochemistry for S-100, with brown stained peripheral nerve tissue $(10 \mathrm{X}))$.

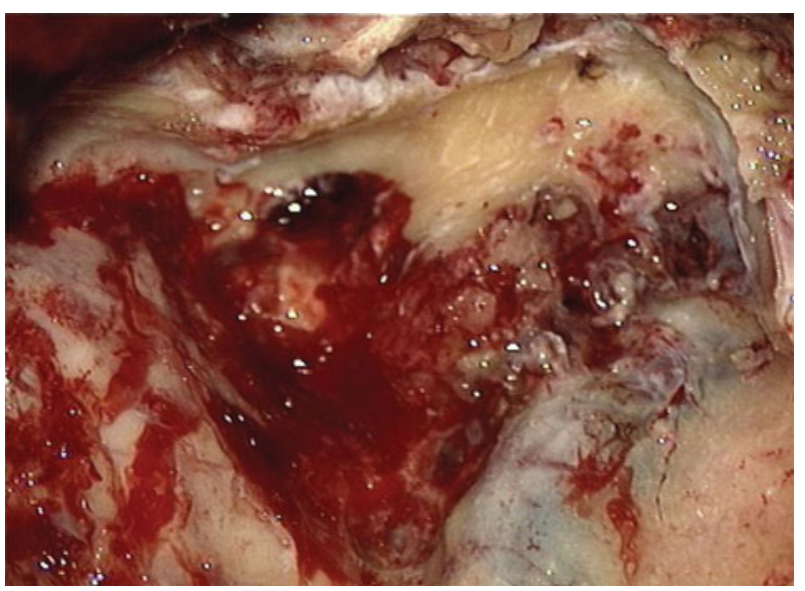

FIG. 4

Macroscopic appearance of the tumour completely filling the mastoid cavity. fibroblast growth factor-23 showed reduced serum phosphorus levels, increased phosphate excretion and features of osteomalacia. ${ }^{5}$ Similarly, most phosphaturic mesenchymal tumours have been found to over-express fibroblast growth factor-23, and thus exhibit clinical features of osteomalacia.

Patients affected by phosphaturic mesenchymal tumours typically exhibit the following clinical features of osteomalacia: bone pain, muscle weakness and fatigue. The biochemical markers include hypophosphataemia, hyperphosphaturia, decreased tubular phosphate reabsorption, and inappropriately normal or low 1,25-dihydroxyvitamin D3. Interestingly, not all patients show clinical or laboratory signs of osteomalacia, one possible explanation being that the tumour secretes inactive or insufficient fibroblast growth factor-23. ${ }^{2}$ Patients may present with signs of the tumour, as in the current case report, wherein the patient initially presented with increasing deafness and then re-presented with recurrent tumour and progressive facial nerve palsy.

A prototypical phosphaturic mesenchymal tumour, mixed connective tissue variant, contains neoplastic cells that are typically spindle to stellate shaped, with low grade nuclear atypia and low mitotic activity. The cells are embedded in a distinctive myxoid to chondromyxoid matrix that often contain foci of 'grungy' or flocculent calcification. Other features of the tumour include osteoclast-like giant cells, mature fat, woven bone and areas of microcystic change. Some morphological variants of the tumour have been described. As in the case described here, a more haemangiopericytoma-like pattern may occur, particularly in tumours of the sinonasal tract. Nevertheless, the immunohistochemical stains for pan-cytokeratin, desmin, S-100 and cluster of differentiation 34 glycoprotein are predominantly negative in the neoplastic cells of all morphological variants. ${ }^{2,6}$

- The phosphaturic mesenchymal tumour, mixed connective tissue variant, is extremely rare

- It is frequently associated with oncogenic osteomalacia

- Most of these tumours are benign; recurrence and metastasis are rare, but can be locally aggressive

- Early diagnosis minimises long-term osteomalacia sequelae and prevents extracranial and intracranial complications

Complete resection of the tumour is the current treatment of choice for phosphaturic mesenchymal tumour, mixed connective tissue variant. The biochemical and clinical signs of osteomalacia tend to normalise within weeks following successful resection of the tumour, and it has been estimated that 90 per cent of patients are cured with complete resection. ${ }^{7}$ Even in histologically malignant tumours, local recurrence or distant metastasis is extremely rare. ${ }^{2}$ Of the reported cases in which radiotherapy was used post-resection to treat malignant phosphaturic mesenchymal tumours, mixed connective tissue variant, only two showed limited success. It is hypothesised that the capacity of the tumour to produce fibroblast growth factor-23 might be altered by radiotherapy. ${ }^{8}$ In the case reported here, although the tumour appeared histologically benign, it showed an aggressive behaviour; thus we offered the patient radiotherapy post-operatively, which she refused. 


\section{Conclusion}

The phosphaturic mesenchymal tumour, mixed connective tissue variant, is a very rare neoplasm. This is the first reported case of the tumour involving the temporal bone. This case report highlights the occurrence of this entity, in an effort to raise its profile amongst clinicians and pathologists. Further similar cases need to be reported in the literature in order to understand the tumour's associated clinical behaviour and to determine the best modality with which to treat this tumour.

Although most reported cases are benign and slowgrowing, they can be aggressive and recurrent, as this case report demonstrates.

Early recognition of the condition remains of the utmost importance to minimise the debilitating consequences of long-term osteomalacia in affected patients, and to prevent extracranial and intracranial complications caused by the tumour.

\section{References}

1 Weidner N, Santa Cruz D. Phosphaturic mesenchymal tumors. A polymorphous group causing osteomalacia or rickets. Cancer 1987;59:1442-54

2 Folpe AL, Fanburg-Smith JC, Billings SD, Bisceglia M, Bertoni F, Cho JY et al. Most osteomalacia-associated mesenchymal tumors are a single histopathologic entity: an analysis of 32 cases and a comprehensive review of the literature. Am J Surg Pathol 2004;28:1-30

3 Shimada T, Mizutani S, Muto T, Yoneya T, Hino R, Takeda S et al. Cloning and characterization of FGF23 as a causative factor of tumor-induced osteomalacia. Proc Natl Acad Sci U S A 2001;98:6500-5

4 Jonsson KB, Mannstadt M, Miyauchi A, Yang IM, Stein G, Ljunggren $\mathrm{O}$ et al. Extracts from tumors causing oncogenic osteomalacia inhibit phosphate uptake in opossum kidney cells. $J$ Endocrinol 2001;169:613-20

5 Shimada T, Hasegawa H, Yamazaki Y, Muto T, Hino R, Takeuchi Y et al. FGF-23 is a potent regulator of vitamin D metabolism and phosphate homeostasis. J Bone Miner Res 2004;19:429-35

6 Woo VL, Landesberg R, Imel EA, Singer SR, Folpe AL, Econs MJ et al. Phosphaturic mesenchymal tumor, mixed connective tissue variant, of the mandible: report of a case and review of the literature. Oral Surg Oral Med Oral Pathol Oral Radiol Endod 2009;108:925-32

7 Reyes-Múgica M, Arnsmeier SL, Backeljauw PF, Persing J, Ellis B, Carpenter TO. Phosphaturic mesenchymal tumor-induced rickets. Pediatr Dev Pathol 2000;3:61-9

8 Uramoto N, Furukawa M, Yoshizaki T. Malignant phosphaturic mesenchymal tumor, mixed connective tissue variant of the tongue. Auris Nasus Larynx 2009;36:104-5

Address for correspondence:

Mr M I Syed,

Department of Otolaryngology,

The Royal Infirmary,

Edinburgh E16 4SA,

Scotland, UK

Fax: +44 (0)131 5363757

E-mail: iqbalms@hotmail.com

Mr M I Syed takes responsibility for the integrity of the content of the paper

Competing interests: None declared 